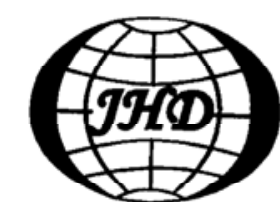

www.sciencedirect.com/ science/journal/10016058

\title{
Call For Paper \\ 5th International Maritime Conference on DESIGN FOR SAFETY \\ and \\ 4th Workshop on RISK-BASED APPROACHES IN THE MARINE INDUSTRIES
}

\author{
(DFS-2013, November 25-27, 2013, Shanghai, China)
}

In 1999, the first International Maritime Conference on Design for Safety was successfully held in Glasgow, UK as a WEGEMT Euro Conference with the sponsorship of the European Commission. Responding to the expectation from the world maritime safety community, the second was held in 2004 in Japan. The third and fourth were held in USA, 2007 and Italy, 2010, respectively. This year the Design for Safety conference including a single event of the 4th Workshop on Risk-Based Approaches in the Marine Industries will be held in Shanghai on November 2527,2013 . The conference is organized by Shanghai Jiao Tong University and is co-organized/ sponsored by Marine Design and Research Institute of China (MARIC) and China Classification Society (CCS). Under the Key-word of "Design of Safety", the Conference expects positive participation of the people from Administrations, Classification Societies, Universities, Research Institutes, Ship Design Offices, Shipbuilding and Shipping industries, Oil and Gas and any other industry with knowledge-intensive, safetycritical products from all over the world to exchange the latest information and innovative ideas. The Conference welcomes papers in aspects of Design for Ship Safety and topics in the areas of:

Philosophy/Framework on Design for Safety, such as safety culture, active and passive safety, life cycle design, risk-based regulations.

$>$ Methodology/Application on Design for Safety, such as risk analysis, performance-based approach, Formal Safety Assessment, first principles approach, risk-based design.
Ship Stability Safety, such as probabilistic damage stability, flooding analysis and control, intact stability code, high-speed craft, fishing vessel safety.

$>$ Fire/Explosion Safety, such as IMO "Alternative Design and Approaches", fire risk analysis and control.

> Structural Strength, such as bulk carrier/tanker safety and reliability.

$>$ Operational Safety, such as passenger ship evacuation, decision support systems, crew training.

$>$ Ocean Engineering Structures Safety, such as risk assessment of ocean engineering structures, limit state design of offshore structures, risk management in ocean engineering projects.

More updated information of the conference can be found at http://naoce.sjtu.edu.cn/sub/dfs2013/

Conference paper can be submitted to:

DFS-2013 Secretary General

Prof. Dr. Decheng Wan

School of Naval Architecture, Ocean and Civil Engineering

Shanghai Jiao Tong University

Dongchuan Road 800

Shanghai 200240

China

Email: wan_decheng@126.com

Fax: +86213420 5685

Tel: +86213420 5697 